Pediatric Critical Care Resource Algorithms, Worksheet, and Triage Team Guidelines. 5. Discuss lessons learned and future considerations regarding scarce resource allocation.

Background: In a disaster situation when resources are scarce, it's agreed that health care providers have a duty to care, but also a duty to steward resources within an ethical framework. ${ }^{3}$ Operationalizing these ideals is challenging, especially when pediatric critical care itself is limited and specialized. The population of Washington state is 7.1 million, with $22.5 \%$ $<18$ years. ${ }^{4}$ There are only 119 PICU beds in the state, and only $30 \%$ are open at any time. During an overwhelming disaster, the number of critically injured and ill children may exceed resources available in spite of conservation and surge strategies. The NWHRN established the DCAC in 2012 to bring regional clinical leaders together to discuss scarce resource allocation.

Methods: Using a modeling tool developed by the US CDC, we estimated the potential pediatric critical care needs during a pandemic. We then gathered data regarding regional pediatric critical care resources, reviewed current literature, discussed conservation and surge strategies, and developed the Pediatric Critical Care Algorithm, Worksheet, and Triage Team Guidelines.

Results: The King/Pierce County Pediatric Critical Care Triage Algorithm and accompanying Worksheet are to be used with the Triage Team Guidelines. These documents are part of a regional Concept of Operations for Scarce Resource Management Plan.

Conclusion: This presentation outlines our process and provides our regional recommendations for pediatric critical care triage, as well as lessons learned and future recommendations. ${ }^{1,3} \mathrm{IOM}$ reports Crisis Standards of Care 2009. ${ }^{2}$ Kissoon N. Deliberations and recommendations of the Pediatric Emergency Mass Critical Care Task Force: Executive summary. Pediatr Crit Care Med. 2011;12:S103-108. ${ }^{4}$ USA Census. gov est 2015.

Prehosp Disaster Med 2017;32(Suppl. 1):s154-s155

doi:10.1017/S1049023X1700423X

\section{Reunification Toolkit for Community Hospitals: Applying Education Principles to a Real World Problem Deanna Dabl Grove}

Pediatrics, UH Rainbow Babies and Children's Hospital, Cleveland/ $\mathrm{OH} /$ United States of America

Study/Objective: Our State Pediatric Disaster Coalition (PDC) was tasked with addressing community hospitals' concerns regarding reunification of children and parents/guardians in a disaster.

Background: Our PDC has representation from public agencies to hospitals. Many reunification tools currently exist on the internet. There were many new concepts to introduce to this audience of adult learners, the Cognitive Dimensions Chart (CDC) was applied during the creation of the toolkit.

Methods: A website was created to link key materials together. Regional hospital coordinators within our state could access materials for education and assist community hospitals to coordinate efforts with local agencies for reunification.
Results: Upon completion of this four component reunification toolkit, hospital emergency planners and safety personnel will have improved capability and capacity for pediatric patients that are separated from parents or guardians. A reunification plan is a vital part of a comprehensive hospital disaster. The first component creates awareness of facts and understanding of reunification for children and families through personal stories. The second component, a framework, explains a reunification plan and identifies key partners to conceptualize and apply to their unique situation. The third and cornerstone component, a checklist, with a step-by-step approach to creating a reunification plan. Included in this checklist are web linked resources to create procedures and detailed individualized analysis. Finally, a disaster drill narrative with a patient list and scenario are included to create either a live or simulated drill, to be able to test the new plan and create corrective actions to enhance the plan, and allows the learner to bring all the components together using metacognition.

Conclusion: This is a practical educational project, applied to a real-world problem, to benefit community hospital emergency management and safety personnel, in addition to children and families in our state to address the importance of reunification in disasters.

Prehosp Disaster Med 2017;32(Suppl. 1):s155

doi:10.1017/S1049023X17004241

Improving Disaster Preparedness for Children and Families: A National Curriculum for Pediatric Emergency Medicine

\section{Fellows}

Deanna Dabl Grove ${ }^{1}$, Mark Cicero ${ }^{2}$, Nathan Timm³ Brent Kaziny ${ }^{4}$, Amyna Husain ${ }^{5}$, Stuart Bradin ${ }^{6}$, Marie Lozan ${ }^{6}$

1. Pediatrics, UH Rainbow Babies and Children's Hospital, Cleveland/OH/United States of America

2. Emergency Medicine, Yale New Haven Children's Hospital, New Haven/CT/United States of America

3. Emergency Medicine, University of Cincinnati - Cincinnati Children's Hospital, Cincinnati/OH/United States of America

4. Texas Medical Center, Houston/TX/United States of America

5. John Hopkins Hospital, Baltimore/MD/United States of America

6. Department Of Emergency Medicine, University of Michigan, Ann Arbor/MI/United States of America

Study/Objective: Disaster Preparedness (DP) training is limited within Pediatric Emergency Medicine (PEM) fellowships. Background: A survey of PEM fellowship directors revealed that $70 \%$ did not incorporate a disaster preparedness curriculum. Disasters can occur in any region and pediatric emergency medicine physicians play a key role in the response. A standard disaster preparedness curriculum is a necessary component of any pediatric emergency medicine fellowship. A nationallybased curriculum has the advantage of providing a common knowledge base for physicians in training that can be expanded and elaborated for individuals and programs.

Methods: PEM experts in disaster preparedness reviewed the curriculum competencies proposed by National Center for Disaster Medicine and Public Health (June 2013), and Entrustable Professional Activities (EPA) for PEM physicians related to disaster preparedness. Comparison of these 